\title{
Towards a realistic simulation of plate margins in mantle convection
}

\author{
Shijie Zhong ${ }^{1}$ \\ Department of Geological Sciences, University of Michigan, Ann Arbor \\ Michael Gurnis \\ Seismological Laboratory, California Institute of Technology, Pasadena
}

\begin{abstract}
We have incorporated faults as plate margins into time-dependent mantle convection models by using a mixed Eulerian and Lagrangian finite element formulation. Plate margins in our models can migrate dynamically in response to variations in mantle buoyancy, and this enables us to study the dynamics of mantle and plates including changes in plate size. Convection models in a cylindrical geometry with such a faulted converging margin, non-Newtonian rheology, and continents indicate that the converging margin tends to migrate oceanward. The oceanward trench migration leads to an increase in the size of the overriding continental plate.
\end{abstract}

\section{Introduction}

The angular velocity of plates with respect to their Euler poles is nearly constant within a plate, but across plate margins velocity changes abruptly in magnitude and direction. This change in plate velocity ultimately is related to brittle failure and displacement along pre-existing faults. While brittle faulting occurs on relatively short time scales, numerous faulting events and fault planes define the nature of the discontinuity of plate margins on longer geologic time scales. The velocity of an overriding plate near a converging plate margin is predominantly horizontal at depth (Figure 1a), which is rather clear for South American subduction zones where the overriding plate is a non-subducting continent. However, the velocity of a subducting plate must have both significant horizontal and vertical components in the hot spot reference frame in order to achieve subduction (Figure 1a). If velocities of the overriding and subducting plates are resolved on the plate boundary separating the plates, the tangential velocity along the plate boundary must be discontinuous from one plate to another (Figure 1a). This discontinuous tangential velocity reflects the deformation accomplished primarily by thrust-faulting earthquakes. This analysis holds independent of the motion of the overriding plate, as long as subduction is one-sided. Besides having a discontinuity in relative motion, plate margins also migrate with respect to the hot spot reference frame. The migration of plate margins is usually accompanied with changes in plate size.

\footnotetext{
${ }^{1}$ Now at Seismological Laboratory, California Institute of Technology, Pasadena, CA 91125.
}

Copyright 1995 by the American Geophysical Union.
It is necessary to include tectonic plates in models of mantle convection [Davies, 1988; Gurnis and Hager, 1988; Gable, et al., 1991], and we believe that plate margins hold the key to simulating plates properly. Dynamic models of mantle convection have shown that the negligibly small strain-rates within a single plate can be obtained if the effective viscosity of plate margins is substantially smaller than that of the plate interior [Christensen, 1983]. In most previous models, plate margins have been fixed in space and usually only a single plate is incorporated. As a result, they have difficulties in addressing how convection influences plate dynamics, including the evolution of plate size. It is necessary to formulate convection models with realistic plate margins that include the discontinuity in velocity and their mobility.

Tectonic faults have been incorporated into a viscous medium to study long term lithospheric deformation [Barr and Houseman 1992, 1994; Zhong and Gurnis, 1994]. Faults are treated as interfaces which have discontinuous tangential velocity but continuous normal velocity. Dynamic models of subduction zones with a fault representing a converging margin can explain outer rise - trench - back arc basin topography and the covariation of trench depth with age of the lithosphere and dip angles of the fault and slabs [Zhong and Gurnis, 1994]. The basic techniques for incorporating faults as plate margins into time dependent mantle convection models are formulated in this paper.

\section{Implementation of Time Evolution of Faults}

The basic governing equations for mantle convection are mass, momentum, and energy conservation which can be numerically solved using a finite element method [e.g., King, et al., 1990]. The strategy for solving these equations is first to solve the energy equation and update temperature using the velocity at the previous time step and second to solve the momentum equation with an incompressibility constraint and update the velocity using the new temperature.

To solve for the flow when a fault is present is equivalent to solving the momentum equation in the presence of constraints derived from the fault [Zhong and Gurnis, 1994]. There are two constraints resulting from a fault [Barr and Houseman, 1992, 1994; Zhong and Gurnis, 1994]. 1) Across the fault, both normal velocity and normal stress are continuous, but the tangential velocity may be discontinuous. 2) The flow can be decoupled or weakly coupled on either side of the fault, depending on an imposed shear stress on the fault. A Lagrange multiplier method is used to enforce the constraints into the momentum equation. Our techniques are general and can be 


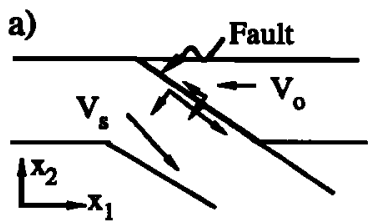

c)

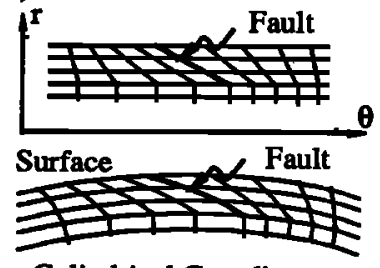

Cylindrical Coordinates b)

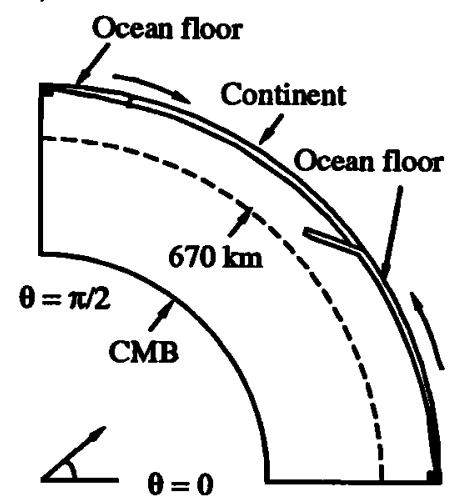

Figure 1. (1a) Typical plate velocity pattern of subduction zones. $V_{o}$ and $V_{s}$ represent overriding and subducting plate velocities, respectively. (1b) Model setup for case 5. (1c) Layouts of finite elements and a fault in two different coordinates.

used to model normal, thrust, or strike-slip faults in two or three dimensions [Zhong and Gurnis, 1994].

In general, the resulting tangential velocity on faults is discontinuous and the normal velocity does not vanish [Barr and Houseman, 1992]. While the discontinuous tangential velocity does not alter fault geometry, the normal velocity implies that the fault must migrate horizontally or change dip with time. In reality, tectonic faults may result from brittle behavior of rocks rather than viscous behavior. Faults migrate in our formulation such that fluid does not "cross" the fault. The horizontal motion of faults is consistent with the observation that plate margins migrate. By incorporating the time evolution of faults, we can study how plates change their geometry in response to convection.

The time-dependence of mantle convection is introduced through the energy equation. In order to accommodate a mobile fault, the procedure of solving the system of equations must be modified. Our procedure is: 1) solve the energy equation and update the temperature field using velocity at the previous time step, 2) update the position of faults using normal velocity of faults from the previous time steps, 3) remesh such that the updated faults are located on mesh nodes and interpolate the updated temperature onto the new mesh, and 4) solve the momentum equation with constraints derived from the newly located fault and update velocity with the updated and interpolated temperature. While an Eulerian formulation is used to solve for temperature and velocity, a Lagrange formulation for tracking faults and remeshing is required. Temperature is continuous on the fault during the solution of the energy equation.

For simplicity, during the process of updating positions of the faults, the faults are constrained to be straight lines and the depth to which the faults extend is assumed constant. A leastsquares method is used to fit the updated positions of faults into straight lines, and the straight lines represent the new faults used in the remeshing. In general, remeshing is computationally expensive, because the finite element stiffness matrix must be reconstructed after remeshing. However, since the stiffness matrix must be reconstructed at the end of each time step in simulating plates when rheology is temperature- and strain rate-dependent, a few remeshings do not result in significant additional computational cost.

\section{Results and Discussion}

We will first present four cases $(1-4)$ to show the basic behavior of faults under the influence of buoyancy forces and then present two cases (5 and 6) with tectonic plates to show how faulted converging margins and plate size evolve with the subduction process. Cases 1 - 4 with constant viscosity are computed with a two dimensional Cartesian box of an aspect ratio 2 with $128 \times 64$ bilinear elements. Cases 5 and 6 with plates are computed in a cylindrical annular geometry with temperature- and strain rate-dependent viscosity. For all the cases, a reflecting boundary condition is applied on the two side walls; on the top and bottom boundaries, free slip and isothermal (if applicable) boundary conditions are used. Zero shear stress is applied on the faults.

\section{Faults Under the Influence of Buoyancy Forces}

Cases 1 and 3 have a different dip angle and case 2 does not include a fault. For these three cases, only the momentum and continuity equations are solved; no time evolution is involved. In the three cases, an identical patch of negative buoyancy is included at the center of the box.

In case 1 , a fault with a dip angle of $45^{\circ}$ and depth of 0.25 is located at $x_{1}=0.875$ on the top boundary (Figure 2a). There are kinks on stream lines across the fault (Figure 2a), reflecting the discontinuity in tangential velocity across the fault (Figure 3a). For comparison, Figure $2 b$ shows stream lines for case 2 which is identical to case 1 but without the fault. The lower plane of the fault has a larger tangential velocity than the upper plane (Figure 3a). The normal velocity on the fault (Figure 3a) indicates that the fault tends to rotate and move toward the symmetry plane of the box. In case 3 , the dip angle and the length of the fault are $90^{\circ}$ and 0.25 , respectively (Figure 2c). Since the fault is aligned with the symmetry plane of the flow system, the fault does not have effect on the flow a

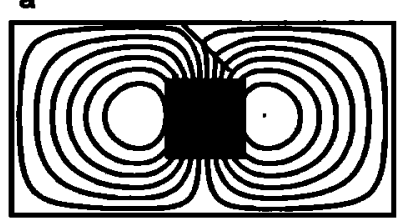

c
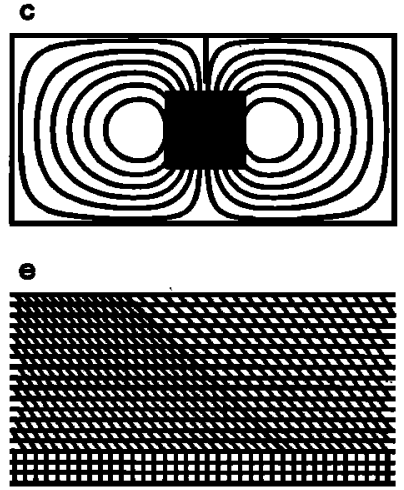

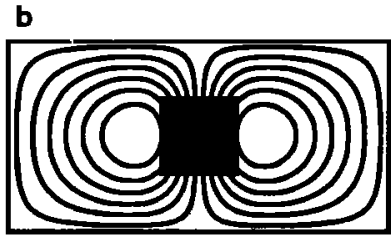

d

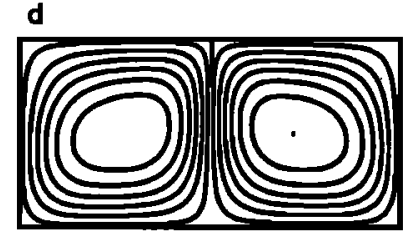

$f$

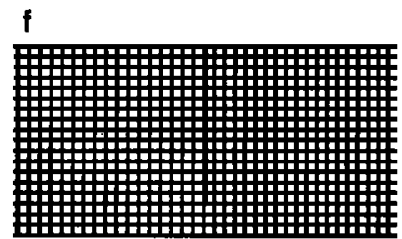

Figure 2. Flow fields for cases 1 (2a), 2 (2b) and 3 (2c), the flow field at the final stage of case 4 (2d), and meshes near the faults for the initial (2e) and final (2f) stages of case 4 . The shaded regions in Figures $2 a, 2 b$, and $2 c$ represent the distribution of buoyancy. The straight lines attached to the top boundaries in Figures $2 \mathrm{a}, 2 \mathrm{c}$, and $2 \mathrm{~d}$ represent fault planes. Faults in Figures $2 e$ and $2 f$ are represented by a thick line. 

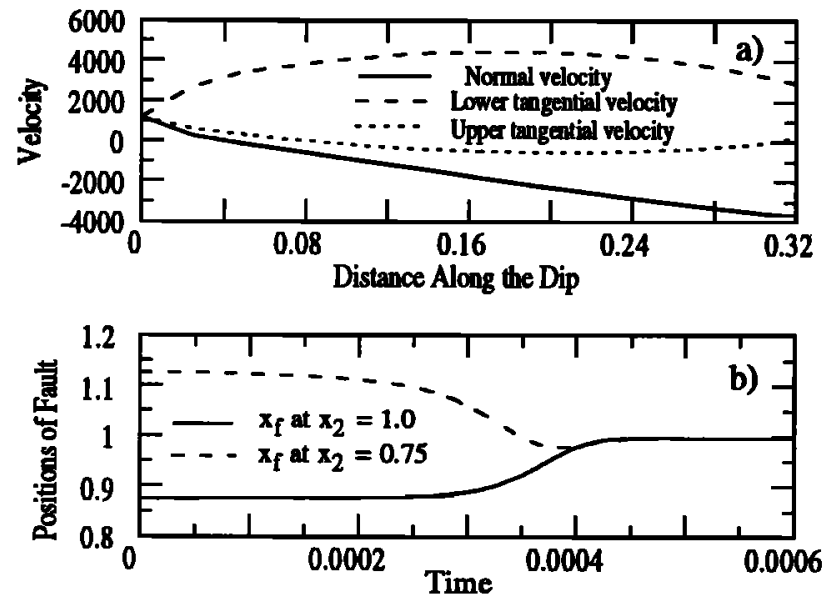

Figure 3. Tangential and normal velocities on the fault plane for case 1 (3a) and the time evolution of the two ends of the fault for case 4 (3b). A positive tangential velocity is defined as along the down dip direction; a positive normal velocity is defined as that with a positive $x_{2}$ component.

and the flow (Figure 2c) is identical to that for case 2 excluding the fault (Figure 2b).

Case 4 is used to study time evolution of a fault. The initial geometry of the fault (Figure $2 e$ ) is identical to that of case 1 (Figure 2a). The initial temperature used bere is given such that two symmetric convection cells would develop with the downwelling limb on the center plane if no fault were present. With this initial condition, the initial $45^{\circ}$ dipping fault becomes progressively vertical and aligned with the center plane (Figure 3b). The final flow field is shown in Figure 2d. Along with the locations of the fault, meshes near the fault at the initial and final stages are shown in Figures $2 e$ and $2 f$, respectively.

Even though we could not confirm our numerical results with analytic solutions for the buoyancy driven faults, we find that for flows with a fault but no body force our fault algorithm yields solutions that compare well with analytic solutions developed by Barr and Houseman [1994] with an error less than $1 \%$.

\section{A Resolution Study with Plates}

Case 5 is computed in a quarter of a cylinder (Figure 1b) to study how plates defined by faults interact with mantle convection. Material properties and dimensions representing the mantle are used for all the parameters (Table 1). A fault with a $30^{\circ}$ dip representing a converging plate boundary is assumed to extend down to the bottom of an overriding plate. The overriding plate is assumed to be a continental plate. Outside of the continent, regions are occupied by ocean floor (Figure 1b). The formulation of the governing equations in a cylindrical geometry was introduced in Zhong and Gurnis [1993]. Similar to Gurnis and Hager [1988], an isothermal interior $\left(1600^{\circ} \mathrm{C}\right)$ with a cooling oceanic plate is included as an initial condition. Initially, no horizontal gradient in temperature is included in the continent which covers the region between the converging margin and a weak margin at $\theta=\pi / 2$ (Figure 1b). A high temperature is initially set at the diverging margin of overriding plate (i.e., the weak margin at $\theta=\pi / 2$ in Figure 1b) to mimic a continental rifting event.

Different rheologies are used for the continent, oceanic plates, and mantle. For the continent and regions below 410 km depth, the viscosity is Newtonian but still temperaturedependent; above $410 \mathrm{~km}$ except within the continent, the viscosity is temperature- and strain rate-dependent. $A$ generalized rheological law is used to describe both Newtonian and non-Newtonian rheologies [Zhong and Gurnis, 1995]. The average effective radial viscosity structure (Table 1 ) is similar to that inferred from geoid studies [e.g., Hager, 1990]. A uniformly high viscosity is used for the continent (Table 1). While converging margins develop with the temperature- and strain rate-dependent viscosity near the fault, diverging margins are prescribed by weak zones that presumably take into account the weakening by partial melting.

For computational convenience, the fault is assumed to be a straight line in a Cartesian coordinate system for $\boldsymbol{r}$ and $\boldsymbol{\theta}$ in which elements are laid out (Figure 1c); in actuality, the fault is slightly curved toward the surface (Figure 1c). The fault is updated using the normal velocity on the fault. Positions of the continent are updated assuming that the continent has a constant length. For this case with non-Newtonian rheology, iteration on the effective viscosity is needed for obtaining convergent solutions. A mesh with $150 \times 60$ elements which are refined within plates and near faults is used.

The negative buoyancy introduced with the cooling lithosphere yields subduction soon after the model starts. After the subducted slab sinks into the mantle (Figure 4a for temperature and flow at time $A$ ), both subducting and overriding plates increase in velocity (Figure 4c). Surface velocity (Figure 4a) indicates "plate-like" velocity and that overriding and subducting plates move towards each other. As the continent moves oceanward along with the converging margin (trench), the overriding plate is enlarged due to addition of the ocean floor vacated by the continent, but the subducting plate shrinks in size (Figure 4a). Although the enlarged overriding plate consists of both continental and oceanic parts, they form a coherent plate, as indicated by the surface velocity (Figure 4a). As the slab further enters the mantle, the overriding plate velocity starts to decrease synchronously with the decrease in the rate of trench migration. The subducting plate velocity, however, keeps increasing during this period (Figure $4 \mathrm{c}$ ). At time B, the slab has reached the bottom boundary with a steep dip (Figure 4b), and the overriding plate has been enlarged by about $1200 \mathrm{~km}$, the same as the amount of trench migration (Figure 4c). While the velocities on the continent side are predominantly horizontal, on the subducting side the vertical and horizontal

Table 1. Physical Parameters for Cases 5 and 6

\begin{tabular}{lc}
\hline \multicolumn{1}{c}{ Parameter } & value \\
\hline Thickness of the fluid layer, $D$ & $2.9 \times 10^{6} \mathrm{~m}$ \\
Outer radius, $R_{o}$ & $6.37 \times 10^{6} \mathrm{~m}$ \\
Temperature contrast, $T_{b}-T_{o}$ & $1600 \mathrm{~K}$ \\
Reference density, $\rho_{0}$ & $3.3 \times 10^{3} \mathrm{kgm}^{-3}$ \\
Thermal conductivity & $3.0 \mathrm{Wm}^{-1} \mathrm{~K}^{-1}$ \\
Thermal diffusivity, $\mathrm{K}$ & $10^{-6} \mathrm{~m}^{2} \mathrm{~s}^{-1}$ \\
Acceleration of gravity, $g$ & $10 \mathrm{~m} \mathrm{~s}^{-2}$ \\
Thermal expansion, $\alpha$ & $2.0 \times 10^{-5} \mathrm{~K}^{-1}$ \\
Viscosity of the continent & $2.0 \times 10^{25} \mathrm{~Pa} \cdot \mathrm{s}$ \\
Average effective viscosity & \\
in the lower mantle (below $670 \mathrm{~km})$ & $-2 \times 10^{22} \mathrm{~Pa} \cdot \mathrm{s}$ \\
in the transition zone $(410-670 \mathrm{~km})$ & $-4 \times 10^{21} \mathrm{~Pa} \cdot \mathrm{s}$ \\
in the upper mantle $(120-410 \mathrm{~km})$ & $-4 \times 10^{20} \mathrm{~Pa} \cdot \mathrm{s}$ \\
in the oceanic lithosphere (above $120 \mathrm{~km})$ & $-2 \times 10^{23} \mathrm{~Pa} \cdot \mathrm{s}$ \\
\hline
\end{tabular}




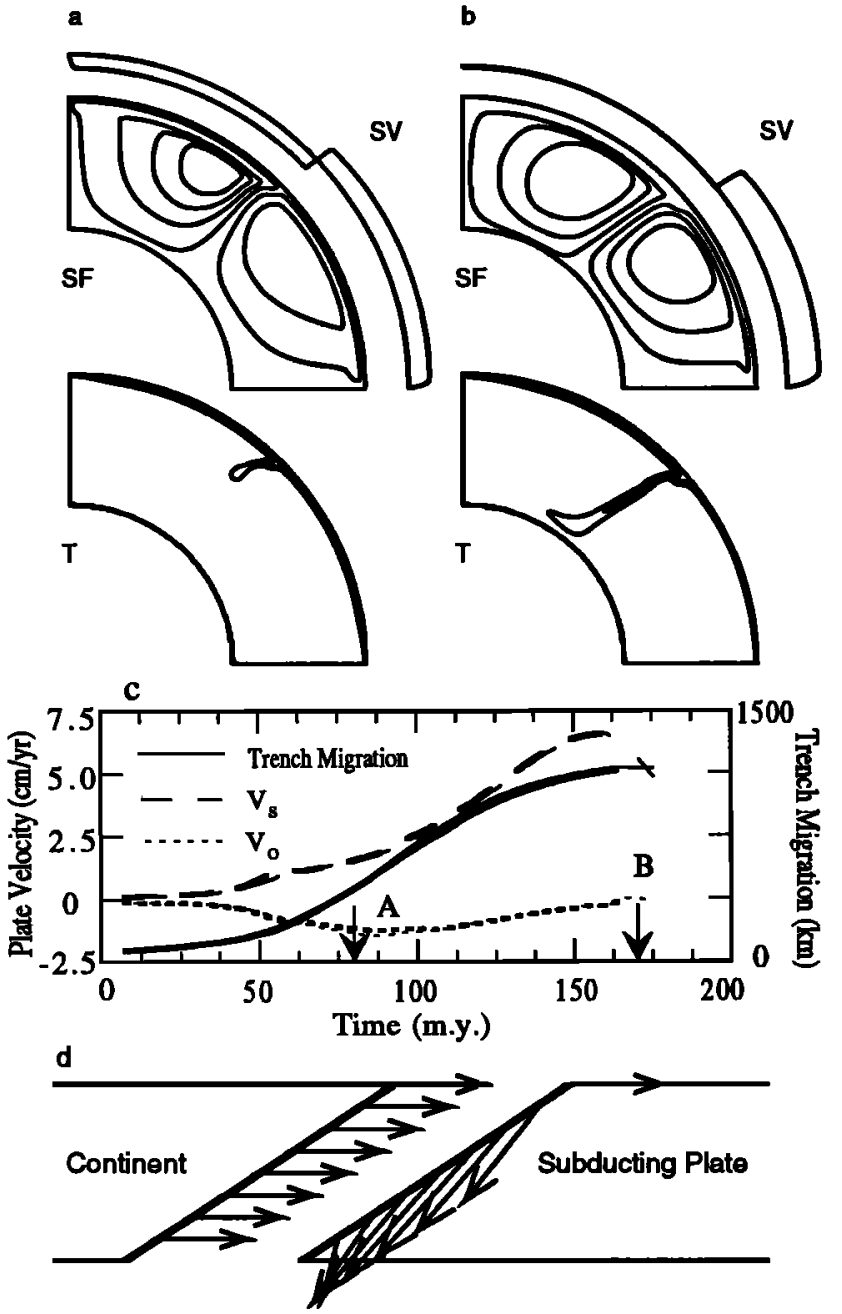

Figure 4. Temperature (T), stream function (SF), and surface velocity (SV) for case 5 at times (4a) A and (4b) B; (4c) time history of overriding $\left(V_{0}\right)$ and subducting $\left(V_{s}\right)$ plate velocities and trench migration for case 5 (thin lines) and 6 (thick lines); and (4d) velocity on the two sides of the fault for the flow field in Figure 4a.

components of velocities are comparable to each other (Figure 4d), indicating an unsymmetric subduction. Since normal velocities do not vary significantly along the fault (Figure 4d), the fault in case 5 does not change its dip significantly with time while migrating.

Case 5 with realistic plate margins and rheology reveals many features of plate tectonics including migrating plate margins and the resulting variations in plate sizes. In order to assure that the dynamics from case 5 are not influenced by frequent remeshing and the moving of material boundaries, a resolution study (case 6 ) has been performed. Case 6 is identical to case 5 but with $250 \times 60$ elements. The time evolution of plate velocities and trench migration from case 6 is almost identical to those from case 5 (Figure $4 c$ ) with a relative difference of plate velocities less than $1 \%$ between cases 5 and 6 at the end of the model run, indicating that case 5 has sufficient resolution and that the algorithm is stable.

\section{Conclusion}

We have incorporated faults as plate margins into timedependent mantle convection models with a mixed Eulerian and Lagrangian finite element formulation. The faults are represented as interfaces on which normal velocity and normal stress are continuous but tangential velocity may be discontinuous. Plate margins defined with such faults can move dynamically in response to mantle flow. Mantle convection models with multiple plates can be formulated by incorporating faulted plate margins and rheology, and these techniques enable us to study the dynamics of the mantle and plates. Convection models in a cylindrical geometry with such a faulted converging margin show that the converging margin tends to migrate oceanward. The oceanward trench migration results in an increase in the size of the overriding plate.

Acknowledgment. We would like to thank G. Houseman for suggesting the comparsion with the analytic solution. Funded by the David and Lucile Packard Foundation and NSF grant EAR-9496185. This is Contribution 5431 of the Division of Geological and Planetary Sciences of the California Institute of Technology.

\section{References}

Barr, T. D., and G. A. Houseman, Distribution of deformation around a fault in a non-linear ductile medium, Geophys. Res. Lett., 19, 11451148, 1992.

Barr, T. D., and G. A. Houseman, Deformation fields around a fault embedded in a non-linear ductile medium, submitted to Geophys. $J$. Int., 1994.

Christensen, U. R., Convection in a variable-viscosity fluid: Newtonian versus power-law rheology, Earth Planet. Sci. Lett., 64, 153-162, 1983.

Davies, G. F., Role of the lithosphere in mantle convection, J. Geophys. Res., 93, 10451-10466, 1988.

Gable, C. W., R. J. O'Connell, B. J. Travis, Convection in three dimensions with surface plates: Generation of toroidal flow, $J$. Geophys. Res., 96, 8391-8405, 1991.

Gurnis, M., and B. H. Hager, Controls on the structure of subducted slabs, Nature, 335, 317-322, 1988.

Hager, B. H., The viscosity profile of the mantle: a comparison of models on postglacial and convection time scales (abstract), Eos Trans. AGU, 71, 1567, 1990.

King, S. D., A. Raefsky, and B. H. Hager, ConMan: Vectorizing a finite element code for incompressible two-dimensional convection in the Earth's mantle, Phys. Earth Planet. Inter., 59, 195-207, 1990.

Zhong, S., and M. Gurnis, Dynamic feedback between an nonsubducting raft and thermal convection, J. Geophys. Res., 98, 12219-12232, 1993.

Zhong, S., and M. Gurnis, Controls on trench topography from dynamic models of subducted slabs, J. Geophys. Res., 99, 15683-15695, 1994.

Zhong, S., and M. Gurnis, Mantle Convection with Plates and Mobile, Faulted Plate Margins, Science, in press, 1995.

M. Gurnis and S. Zhong, Seismological Laboratory, California Institute of Technology, Pasadena, CA 91125.

(Received August 1, 1994; revised November 15, 1994; accepted February 6, 1995.) 